\title{
A Data Transmission Algorithm Based on Dynamic Grid Division for Coal Goaf Temperature Monitoring
}

\author{
Qingsong Hu, ${ }^{1,2}$ Lixin Wu, ${ }^{1,2}$ Fei Geng, ${ }^{1,3}$ and Can Cao ${ }^{1,3}$ \\ ${ }^{1}$ Internet of Things (Perception Mine) Research Center, China University of Mining and Technology, Xuzhou 221008, China \\ ${ }^{2}$ School of Environment Science and Spatial Informatics, China University of Mining and Technology, Xuzhou 221008, China \\ ${ }^{3}$ School of Information and Electric Engineering, China University of Mining and Technology, Xuzhou 221008, China
}

Correspondence should be addressed to Qingsong Hu; hqsong722@163.com

Received 8 January 2014; Accepted 3 March 2014; Published 3 April 2014

Academic Editor: Xin Wang

Copyright (C) 2014 Qingsong Hu et al. This is an open access article distributed under the Creative Commons Attribution License, which permits unrestricted use, distribution, and reproduction in any medium, provided the original work is properly cited.

\begin{abstract}
WSN (wireless sensor network) is a perfect tool of temperature monitoring in coal goaf. Based on the three-zone theory of goaf, the GtmWSN model is proposed, and its dynamic features are analyzed. Accordingly, a data transmission scheme, named DTDGD, is worked out. Firstly, sink nodes conduct dynamic grid division on the GtmWSN according to virtual semicircle. Secondly, each node will confirm to which grid it belongs based on grid number. Finally, data will be delivered to sink nodes with greedy forward and hole avoidance. Simulation results and field data showed that the GtmWSN and DTDGD satisfied the lifetime need of goaf temperature monitoring.
\end{abstract}

\section{Introduction}

Due to the air leakage behind the working face, residual coal in goaf continuously adsorbs oxygen, resulting in the decrease of the ignition point and increase of coal activation grade during coal mining [1]. If aggregating heat cannot dissipate in time, coal spontaneous combustion may take place. Nevertheless, the widespread use of fully mechanized caving mining technology intensified such problems as the augment in residual coal, enlargement in height of roof falling, and air leakage, which have exacerbated the spontaneous combustion problem. Fortunately, there is a self-thermal process before spontaneous combustion and combustion happens only in the condition that the temperature of coal exceeds the critical temperature. Thereupon, dynamic monitoring to the temperature in goaf will be helpful to distinguish the tendency of spontaneous combustion and confirm the site of self-ignition, which makes it possible to take targeted fire prevention and extinguishing measures.

Some methods of detection and positioning of fire source had been found in literatures, such as gas analysis [2], temperature detection [3], geological radar, radio wave, aeromagnetic exploration, electrical resistivity, and computer simulation [4], which played a guiding role for fire prevention and fire extiguishment. However, these methods were not designed for continuous and realtime monitoring, so they can neither reveal the temporal and spatial variation process nor judge or prejudge the fire source location precisely, let alone making targeted judgment and settlement. As a novel method under the mining perceptional things architecture [5], wireless sensor networks (WSNs) gather some important abilities, such as circumstance-aware and self-organization $[6,7]$, with which the collected temperature data can be transported to the sink nodes in working face. Based on the "three-zone" division of goaf, this paper structured the GtmWSN (coal goaf temperature monitoring WSN) model and the assorted data transmission method, named DTDGD (data transmission based on dynamic grid division). Simulations and field data showed that the GtmWSN model and corresponding transmission method suit the lifetime demand well and boast guiding significance to the deployment, preferences, and data collection for temperature monitoring. To the best our knowledge, it is the first WSN model and transmission method for coal goaf. 


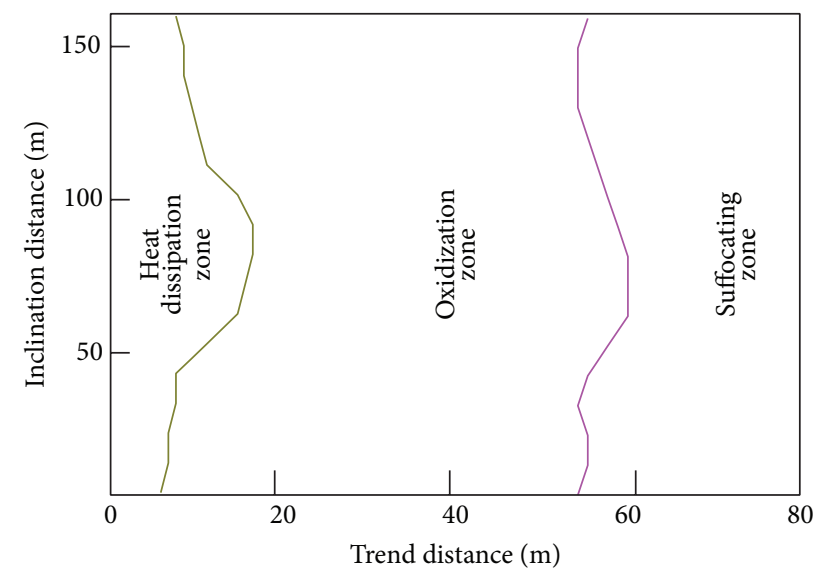

FIgURE 1: “Three-zone” division of coal goaf.

The paper is organized as follows. In the next section, the GtmWSN model and its dynamic features will be presented. Then the data transmission method and its performance simulations under this model can be seen in Sections 3 and 4 , respectively. The last section is a summary.

\section{GtmWSN Model and Its Characteristics}

2.1. The GtmWSN Model. Based on the oxygen level, the coal goaf area can be divided into three zones, named heat dissipation zone, oxidization zone, and suffocating zone, respectively (see Figure 1). For simplicity, we call them HDZ, $\mathrm{OZ}$, and SZ hereafter. Earlier researches showed that $[8,9]$, from the beginning of working face to goaf, these zones are in the range of $0 \sim 10 \mathrm{~m}, 10 \sim 70 \mathrm{~m}$, and over $70 \mathrm{~m}$ separately. Spontaneous combustion will not happen in the HDZ since the fallen roof stones are loosely accumulated, with large void space and air leakage level, and heat generated by coal oxidation will be emited in time. Similarly, the SZ will not combust automatically either, as the fallen rocks have been compacted which fails to maintain the oxidation for spontaneous combustion. However, self-ignition may happen at the OZ which lies between the HDZ and SZ, for heat will continuously accumulate. Thereupon, close attention and emphasis should be paid to the temperature here.

Temperature monitoring nodes are mounted on the hydraulic supports at some intervals, and most of these nodes can fall to the ground easily when the hydraulic supports move forward and self-organize themselves into a coal goaf temperature monitoring WSN (GtmWSN, see Figure 2). Some reinforcement and protection actions should be taken to prevent damage from the roof rocks. Besides, part nodes are fixed (e.g., will not fall) onto the supports evenly spaced and serve as sinks for temperature collection, sending the collection results to AP (access point) by multihop between each other and further to ground monitor servers via industrial Ethernet.

To simplify the theoretical analysis, the dividing lines between different zones are assumed straight lines rather than curves in Figures 1 and 2, and the HDZ, OZ, and SZ lie in

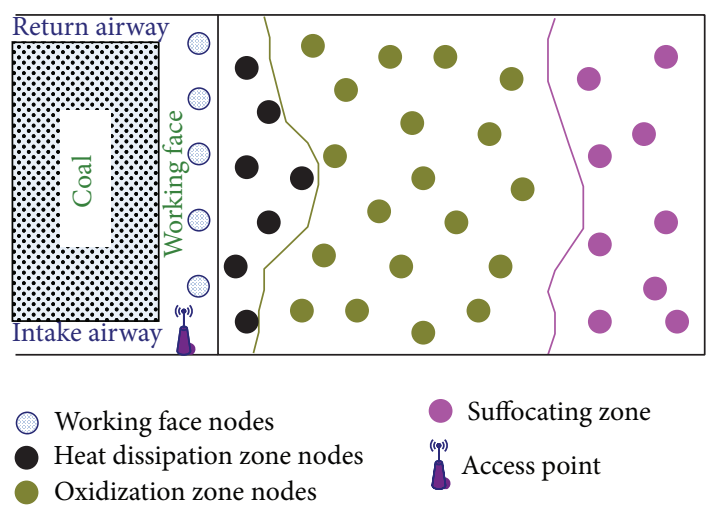

FIgURE 2: Temperature monitoring WSN model of coal goaf.

$(0, p] \mathrm{m},(p, q] \mathrm{m}$, and $(q, r] \mathrm{m}$, respectively. The temperature data collection in SZ will not be taken into consideration for spontaneous combustion will not happen here. Although the HDZ will not combust simultaneously either, the nodes here are relays for OZ data. Thereupon, GtmWSN is a $q \times l$ rectangle network ( $l$ is the length of working face), which is divided into two parts $p \times l$ and $(q-p) \times l$ corresponding to heat dissipation zone and oxidizing zone, respectively.

2.2. The Characteristics of GtmWSN. Comparing to the common WSN [10], the GtmWSN has many characteristics.

(1) The power of GtmWSN nodes is nonrenewable; thereupon, the efficiency of energy consumption must be given full consideration. The sink nodes, however, can get power from lighting cable in the working face, and the energy consumption problem can be left out.

(2) Spontaneous combustion may take place at any part of autoignition area, thus every node deployed in this zone has an equal importance and should not be easily replaced by others.

(3) With the gathered data always heading to working face, the nodes that are near to working face will not only transfer their own data, but also relay data from others, so their energy consumption is much larger.

(4) GtmWSN is a multisink network; therefore, the transmission task is done once a sink has gotten the collected data, which means the GtmWSN is an any cast-oriented network $[11,12]$.

When hydraulic supports move forward, some premounted nodes will fall into heat dissipation zone and become HDZ nodes (called nodes join, see Figure 3). Part nodes in HDZ will enter into $\mathrm{OZ}$ and become $\mathrm{OZ}$ nodes, called role shift. Given the ignorance of temperature monitoring in SZ, all the nodes that enter into this zone no longer belong to GtmWSN, called nodes leave.

Therefore, in forms of identity, role, and relayed data quantity, GtmWSN nodes will change in a dynamic way, see Figure 4. To adapt to this situation, all nodes must perceive to which zone they belong and adjust their corresponding 


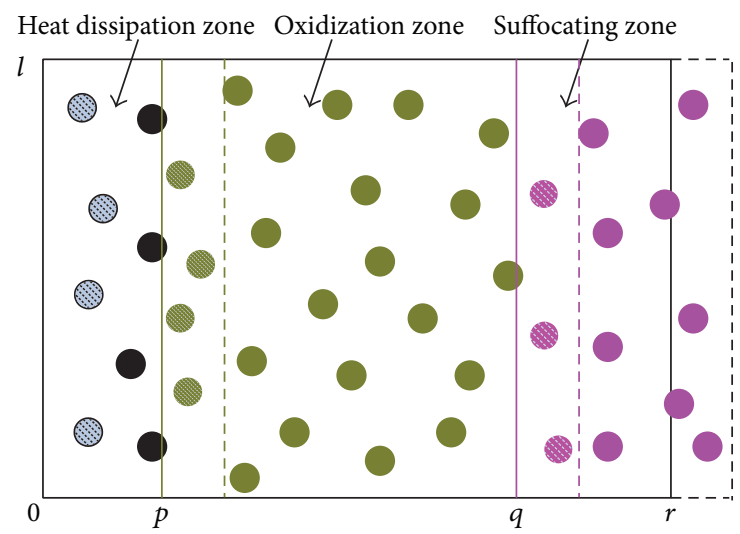

New nodes of HDZ

OZ/SZ transformation nodes

FIgURE 3: Nodes join, leave, and role shift.

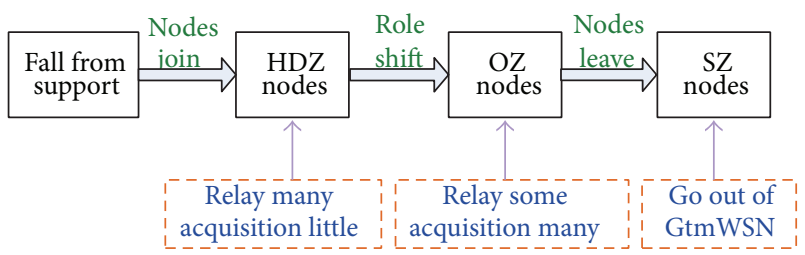

FIgURE 4: Dynamic changes of roles and tasks of nodes.

role. Obviously, common methods aiming at constant and homogeneous WSN cannot meet these demands.

\section{The Data Transmission Scheme of GtmWSN}

This section raises a data transmission scheme named data transmission based on dynamic grid division, briefly called DTDGD, which assumes two work modes, normal mode and abnormal mode. Firstly, the whole network is split into some grids; then each node finds the affiliation grid according to the identifier number (ID) and, hereafter, transmits the gathered data to sinks with greedy relay and hole avoidance.

3.1. Data Acquisition Modes. Suppose the distances between sink nodes are equal, denoting by $d_{s}$. Let the sink node set as $V_{S}$ and the node set of $\mathrm{HDZ}$ and $\mathrm{OZ}$ as $V_{T}, V_{\mathrm{O}}$, respectively. The GtmWSN set is composed of $V_{T}$ and $V_{O}$ nodes, denoting by $V_{G}$ (i.e., $V_{G}=V_{T}+V_{O}$ ).

At beginning, $V_{G}$ nodes are in normal mode and the temperature acquisition cycle is $T_{1}$, and they will send once every $\zeta$ gathering to reduce data size and energy consumption. When the data gathered by $V_{G i}\left(V_{G i} \in V_{G}\right.$, $i=1,2, \ldots,\left|V_{G}\right|$, and $\left|V_{G}\right|$ is the number of $\left.V_{G}\right)$ exceed the temperature threshold, they will switch to abnormal mode, sending once at a gathering to guarantee the data security and shortening the gathering interval to $T_{2}\left(T_{2}<T_{1}\right)$ to monitor the temperature intensively in the exception region. At the same time, the exact and nearby nodes of warning spot will cooperate to get better and more data from focus observation.

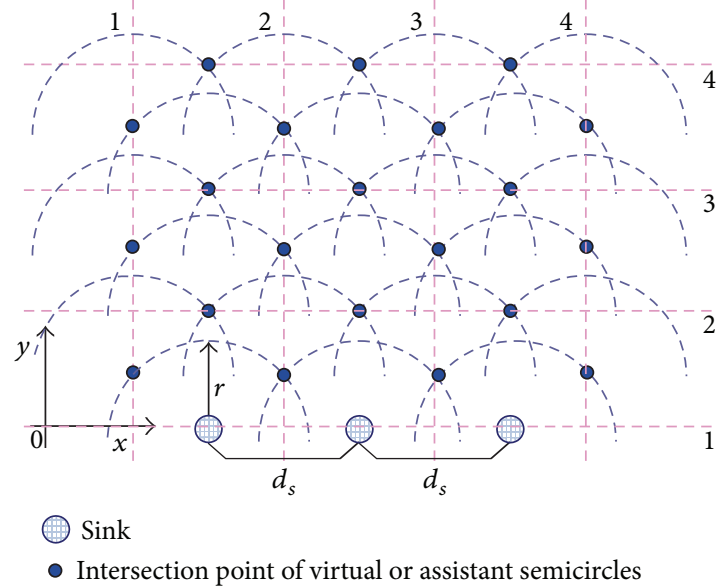

FIGURE 5: Dynamic grid division.

Assume the coordinates of $V_{G}$ nodes are known (the determination method will be described in Section 3.2), but there is no assumption about whether one node knows location of other nodes or not. Besides, the $V_{G}$ nodes are aware of their move direction, which makes it possible to design a greedy data transmission scheme [13].

3.2. Dynamic Grid Division. Taking the elements of $V_{S}$ set as centers and $r$ as radius, some virtual semicircles can be drawn, called the first layer virtual semicircle (see Figure 5). To ensure there is a point of intersection between adjacent semicircles, $r$ must be greater than or equal to $d_{s} / 2$. After that, two assistant points of intersection are added at the leftmost and rightmost semicircles, respectively, whose height is equal to other intersections. Taking these intersection points and assistant intersections as centers and $r$ as radius, the second layer virtual semicircles can be plotted. Similarly, the virtual semicircles of layer $i, i \in N$ can be drawn continuously ( $N$ is a set of positive integers); see Figure 5.

After getting the virtual semicircles, some vertical lines and horizontal lines can be plotted, which contain points of intersection lying in odd layers (and assistant intersections) and points of intersection lying in even layers (and sink nodes), respectively; see Figure 5. These vertical lines and horizontal lines divide GtmWSN into grids with equal area (except for boundary grids), and this process is called grid division. In spite of the different areas, the leftmost and rightmost grids will transmit data correctly, and more information will be presented at Section 3.3. After a whole ploughing, all the hydraulic supports and their accessory nodes make a complete advance; then the network must be redivided. Consequently, the grid division is a dynamic process, which not only ensures the grids timeliness, but also avoids $V_{G}$ nodes fixed on some certain grids all the time and avoid monitoring absence.

Taking the horizontal line containing $V_{S}$ nodes as horizontal axis, positive direction is from left to right and the leftmost in the monitoring network as the origin of coordinate system; the forward direction of vertical axis is from bottom 
to up. Numbering the horizontal lines $1,2, \ldots, i, \ldots, m$ from bottom to top and the vertical lines $1,2, \ldots, j, \ldots, n$ from left to right, we can get the ID of the grid at the top left corner of intersection of horizontal line $i$ and vertical line $j,(i, j)$. For the special case of the rightmost grid, we number it $(i, n+1)$.

Except for the boundary grids, the width (horizontal length) of any other grids is $d_{s}$, which is already known, but the height (vertical length), $h$, must be computed. For this purpose, four nodes are taken out from Figure 5 to make a new figure shown in Figure 6; they are the left and middle sink nodes, named Sink1 and Sink2 here, and the second intersection point $(1,2)$ in the first layer virtual semicircle and the first intersection point $(2,1)$ in the second layer of virtual semicircle:

$$
\begin{aligned}
h & =2 \cdot r \cos \theta \\
& =2 r \sqrt{1-\left(\frac{d_{s}}{2 r}\right)^{2}}=\sqrt{4 r^{2}-d_{s}^{2}} .
\end{aligned}
$$

After the division of grids, $V_{G}$ nodes will find to which grid they belong [14]. As can be seen from Section 2 and Figure 5, the locations of hydraulic supports are known thus the coordinates of $V_{S}$ nodes can be known too. Assuming the coordinate of $i$ th sink node is $\left(x_{i}, 0\right) i \in 1,2, \ldots,\left|V_{S}\right|$, and the $\left|V_{S}\right|$ is the number of $V_{S}$ nodes, we can get the ordinate value set of horizontal line:

$$
l_{m}=\{h, 2 h, \ldots, i h, \ldots, m h\} .
$$

Similarly, we can get the abscissa value set of vertical lines:

$$
l_{n}=\left\{x_{1}-\frac{d_{s}}{2}, \ldots, x_{i}-\frac{d_{s}}{2}, \ldots, x_{n}-\frac{d_{s}}{2}, x_{n}+\frac{d_{s}}{2}\right\} \text {. }
$$

The $V_{S}$ nodes will transmit the information of $l_{m}$ and $l_{n}$ to $V_{G}$ nodes with flooding methods, and $V_{G}$ nodes can determine their own affiliation grids and corresponding grid ID, $(V(i), V(j))$, in accordance with their own coordination $(x, y)$ :

$$
\begin{aligned}
& V(i)= \begin{cases}n+1, & \text { if } x \geq \max \left(l_{n}\right) \\
1, & \text { if } x \leq \min \left(l_{n}\right) \\
n i, & \text { if } l_{n i}<x<l_{n i+1}\end{cases} \\
& V(j)=\frac{\min \left(y \geq l_{m}\right)}{h} .
\end{aligned}
$$

Here, $l_{n i}$ and $l_{n i+1}$ are the $i$ th and $(i+1)$ th elements of $l_{n}$ set, respectively.

Obviously, the coordinate $(x, y)[15]$ of $V_{G i}$ node have to be known to make sense of formula (4). As mentioned earlier, the $V_{G}$ nodes come (fall) from the hydraulic supports, so their horizontal coordination is equal to the hydraulic supports (there might be deviation, but it will not be large and can be overlooked or ignored), which means the value of $x$ is known. In order to determine the value of $y$, a timer will be set up, starting while $V_{G}$ nodes are falling down. If the speed of hydraulic supports is $v$, then the vertical coordination of $V_{G i}$ at moment $t$ is $y=v t$. It is worth noting that the timer can not only indirectly provide the coordinates of $V_{G}$ nodes,

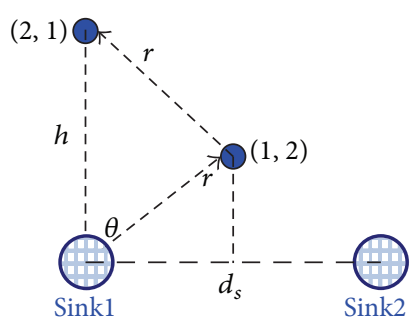

Figure 6: Computation of grid height.

but also the timestamp of temperature data to do time-space analysis of temperature variation in goaf [16].

During period of temperature monitoring, once $V_{G}$ nodes obtain an update command from $V_{S}$, they will recount the current affiliation grids with formula (4). Then the grid IDs will be exchanged with HELLO packet between $V_{G}$ nodes to learn to which grids they belong (current, left neighbor, right neighbor, or sink directional grids), with which greedy relay and hole avoidance can be conducted using the method presented in Section 3.3.

It is noticeable that drawing virtual semicircles in the process of grid division is to determine $l_{m}$ and $l_{n}$ and then help $V_{G}$ nodes confirm the affiliation grids. Nevertheless, there is no need to draw semicircles in practical arithmetic at all because it can get $l_{m}$ and $l_{n}$ in the assistance of horizontal coordination $x_{i}$ of $V_{S}$, grid height $h$, and distance interval $d_{s}$ between sinks. The only reason why semicircles are drawn in Figure 5 is to state the algorithm motivation and the source of $l_{m}$ and $l_{n}$. When the grid size changed, all $V_{S}$ only need to substitute the new $h$ value into formula (4) to calculate out new $l_{m}$ ( $l_{n}$ is only dependent on $x_{i}$ and $d_{s}$, which keep unchanged after initialization) and send the new $l_{m}$ results to $V_{G}$ nodes by flooding means. Based on this feature, no great complexity and expenses exist in the dynamic grid division.

3.3. Data Transmission Based on Dynamic Grid Division. After being divided into grids, the monitoring network adopts greedy method to deliver data $[17,18]$; that is, the nodes in grid $(i, j)$ send their data to nodes in grid $(i-1, j)$ and gradually move close to the sinks. For this purpose, the nodes of adjacent grids must be pairwise connected; that is, the radio range must be greater than or equal to the length of diagonal line of the two neighboring grids (see Figure 7):

$$
d_{01} \geq \sqrt{4 h^{2}+d_{s}^{2}} \text {. }
$$

Obviously, equal mark can satisfy this requirement; thus,

$$
\begin{aligned}
d_{01} & =\sqrt{4 h^{2}+d_{s}^{2}} \\
& =\sqrt{4\left(4 r^{2}-d_{s}^{2}\right)+d_{s}^{2}}=\sqrt{16 r^{2}-3 d_{s}^{2}} .
\end{aligned}
$$

If all nodes of grid $(i-1, j)$ died (hole appears), the algorithm will execute hole avoidance towards right (called rightward avoidance): the grid $(i, j)$ transmits data to grid $(i, j+1)$ (see Figure 7$)$; then the grid $(i, j+1)$ attempts to 


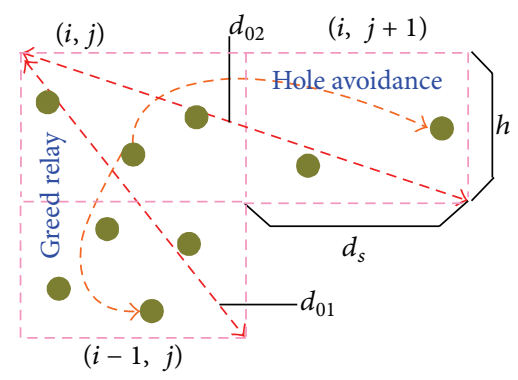

FIgURE 7: Greedy forwarding and hole avoidance based on grid division.

make greedy forwarding; if grid $(i, j+1)$ has judged the grid $(i-1, j+1)$ also as a hole, it relays data to grid $(i, j+2)$, until the rightmost grid fails. If so, similar avoidance method will be executed towards left (called leftward avoidance), starting with the grid $(i, j)$. Apparently, the rightmost and leftmost can only carry out leftward avoidance and rightward avoidance, respectively. During the process of hole avoidance, nodes must ensure the following formula:

$$
\begin{aligned}
d_{02} & =\sqrt{4 d_{s}^{2}+h^{2}} \\
& =\sqrt{4 d_{s}^{2}+\left(4 r^{2}-d_{s}^{2}\right)}=\sqrt{4 r^{2}+3 d_{s}^{2}} .
\end{aligned}
$$

To satisfy the requirements of both greedy forwarding and hole avoidance, one can assume the transmitting power of node is adjustable, making the transmitting distance $d_{0}$ adjust between $d_{01}$ and $d_{02}$. If the transmitting power is fixed, the maximum of $d_{01}$ and $d_{02}$ will be taken to cover neighboring grids completely:

$$
d_{0}=\max \left(\sqrt{16 r^{2}-3 d_{s}^{2}}, \sqrt{4 r^{2}+3 d_{s}^{2}}\right) .
$$

Another approach to realize complete coverage under fixed transmitting power is to adjust the radius of virtual semicircle, making $d_{01}=d_{02}$; that is,

$$
\sqrt{16 r^{2}-3 d_{s}^{2}}=\sqrt{4 r^{2}+3 d_{s}^{2}} \text {. }
$$

So, we obtain the following result:

$$
r=\frac{d_{s}}{\sqrt{2}}
$$

Now, we make a full view of the DTDGD algorithm.

Step 1. After the $r$ value is set, $V_{S}$ nodes calculate the grid height $h$ with formula (1); then get $l_{m}$ with formula (2).

Step 2. The $V_{S}$ nodes compute the $l_{n}$ with formula (3) based on their horizontal coordination $x_{i}$ and $d_{s}$, distance interval between $V_{S}$ nodes.

Step 3. The $V_{S}$ nodes broadcast the $l_{m}$ and $l_{n}$ to $V_{G}$ nodes.

Step 4. The $V_{G}$ nodes determine their own grid ID by formula (4) and then exchange the IDs between each other with HELLO message.

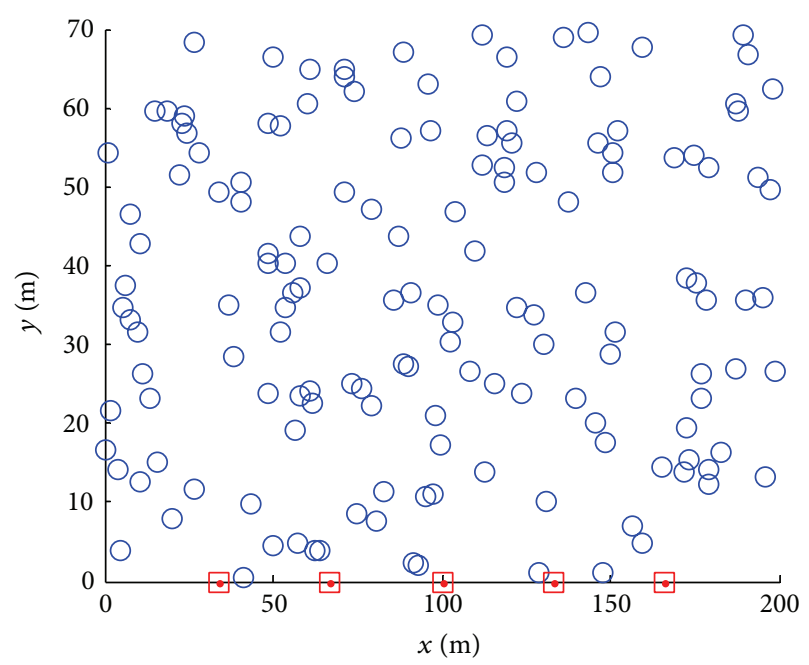

FIGURE 8: Topology of simulation network.

Step 5. The $V_{G}$ nodes decide whether to send their collected data according to working mode. If sent, then greedy forwarding is tried first. If hole exists, hole avoidance is used.

Step 6. After a whole coal cutting in the working face, if there is no need to change the grid height $h$, then a simple update message is flooded to $V_{G}$ nodes. Otherwise, the $l_{m}$ is recalculated using the method described in Step 1 and then flooded to $V_{G}$ nodes, returning to Step 4 .

\section{Performance Evaluations}

The energy consumption model in literature is adopted here [19], with parameter settings of $E_{o}=0.5 \mathrm{~J}, E_{\mathrm{fs}}=10 \mathrm{~nJ}$, and $E_{\text {ele }}=50 \mathrm{pJ}$, representing initial node energy, energy consumption in power amplify circuit, and other circuits, respectively. Supposing 150 nodes are placed in a square area whose $l=200 \mathrm{~m}$ and $q=70 \mathrm{~m}$ and the number of sink nodes NumSink $=5, r=d s / 2+d s / 10$, we get a network like Figure 8 whose blue circles represent $V_{G}$ nodes, and red squares denote sinks. In every acquisition cycle, survival nodes send packets of 200 bits in predetermined time slots. We call it a round when all survival nodes accomplish data collection and sending for once. If one or more survival nodes fail to send data, the network lifetime reaches its end. That is to say, network lifetime is the number of sheaves for survival nodes collecting and sending data normally.

The path loss exponent $n$ has great affection on network lifetime. As it is presented in Figure 9, when $n$ is 2, 3, or 4, the lifetime is 13393,6465 , and 384 rounds, whose dead node number is 24,31 , and 41 , respectively. Therefore, the network lifetime reduces rapidly with the increase of $n$. Without loss of rationality, we assume $n=3$ in the following.

Now we consider the radius of the virtual semicircle, $r$, how to affect the lifetime. Figure 10 describes the network lifetime, under the circumstances of $r=d s / 2+d s / i, i=$ $10,8,6,4$. As can be seen from Figure 10, the lifetime is 


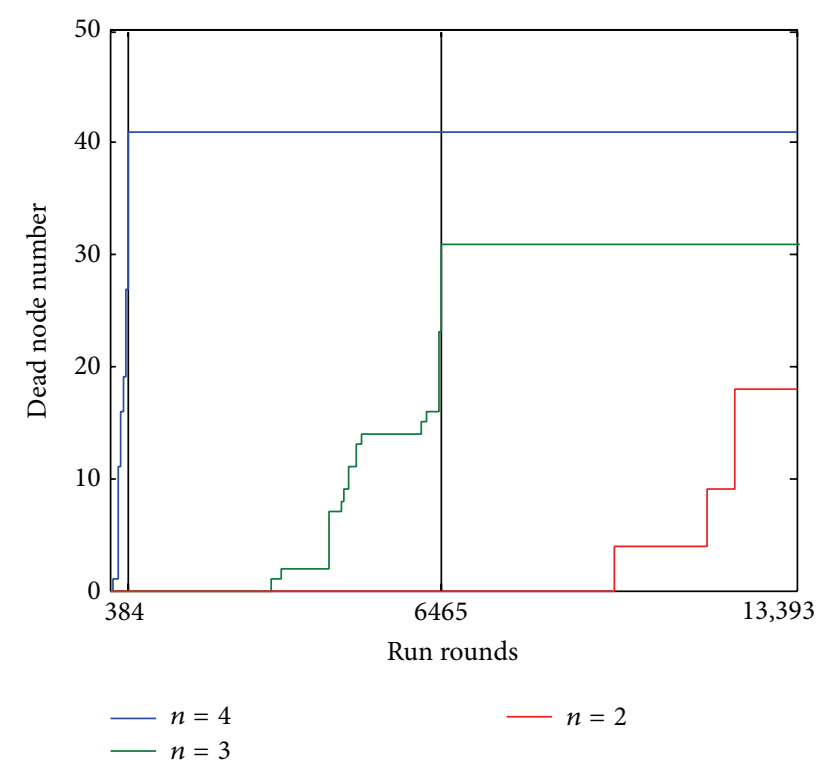

Figure 9: Dead nodes number with different path losses.

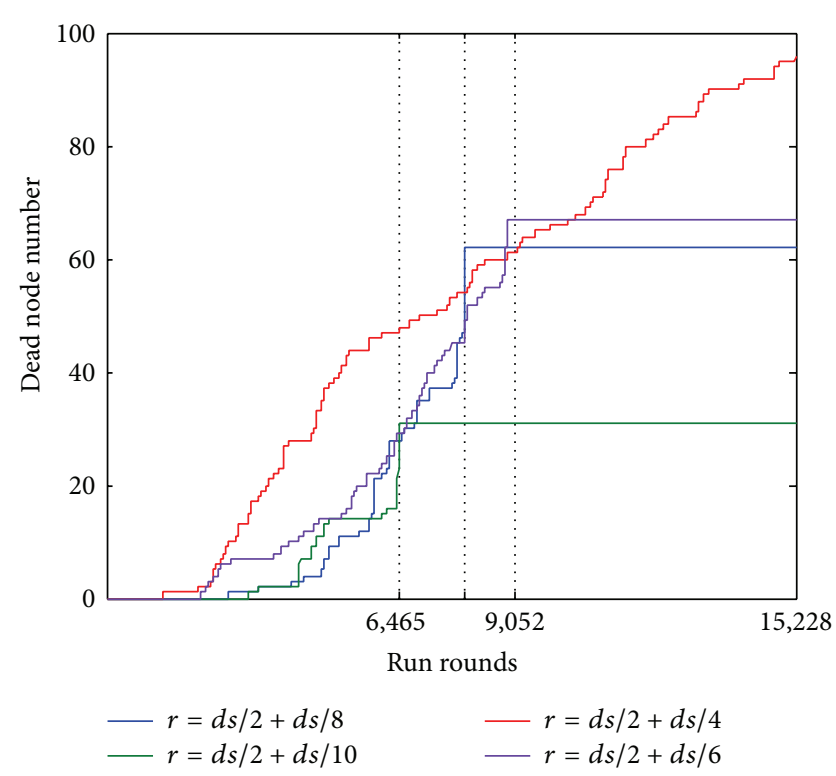

FIGURE 10: Dead nodes number with different radii of virtual semicircle.

$6465,7943,9052$, and 15228 rounds, respectively, and the corresponding dead node number is $31,62,67$, and 96 . That is, there is an incremental impact of radius on network lifetime.

It is noteworthy that the increase of lifetime caused by larger radius may be a false appearance. To demonstrate, the study plots the dead node distribution with the same settings for $r$ as Figure 10, and the result is shown in Figure 11, in which blue circles and red crosses represent survival and dead nodes, respectively. The three parts of Figures 11(a), 11(b), and 11(c) illustrate that under these three $r$ settings, many nodes in oxidization zone have already died and lost monitoring ability. On the contrary, the $r$ setting of Figure 11(d) kept the basic monitoring ability, which proves that it is a reasonable radius setting.

Our simulations found that the sink number, denoted by NumSink, is also an affecting factor of network lifetime, as shown in Figure 12. We can see that under the conditions that NumSink is equal to $3,4,5,6$, or 7 , the lifetime is 4861,6584 , 6465,5443 , and 4076 rounds, respectively. Thus, there must be an optimum NumSink, and here it is 4 .

Summing up the above, we can come to the following conclusions.

(1) In the case of $E_{o}=0.5 \mathrm{~J}$ and $n=3$, GtmWSN is able to reach about 6500 rounds network lifetime with a sense of monitoring. With the advances of battery technology, $E_{o}$ can be set to larger values in the future, which would further improve the life span of the network significantly.

(2) If the acquisition cycle is 10 minutes in normal mode, then only 144 rounds a day are needed, so the network can support more than 40 days of continuous monitoring. The actual advancing distance of working face varies with the differences of coal production, generally greater than or equal to $2 \mathrm{~m}$ /day, and for a goaf with $q=70 \mathrm{~m}$, the upper limit of the sensor lifetime demand is 35 days. Thereupon, the network and correspondent transmitting algorithm can fully meet the actual demand.

(3) Because the data is sent once at every $\zeta$ collecting times in normal mode, the network lifetime will prolong to $\zeta$-fold of abnormal mode. As previously mentioned, GtmWSN has to send every data at each gathering in abnormal mode and network lifetime could meet this demand which has been proved in (2).

In order to further verify these conclusions, actual measurements are conducted using fiber optic temperature sensors [20, 21]. Figure 13 presents the historical temperature data of the No. 1 channel in 10302 working face in 2 hours, and the sampling interval is 30 seconds. The figure reveals that temperature fluctuated around $23^{\circ} \mathrm{C} ; 10$ minutes greater sampling interval can fully meet this requirement. In addition, because the advance speed of the working face is $8 \mathrm{~m} / \mathrm{d}$, 9 days lifetimes can satisfy the requirement of the $70 \mathrm{~m}$ monitoring scope. It proved the conclusions in (2) and (3). Meanwhile, the assumption about $E_{o}$ and $n$ in conclusion (1) basically coincides with reality and $E_{o}$ can be even bigger; therefore, the conclusion of (1) has been proved as well.

\section{Conclusions}

As an important method of the continuous and real-time monitoring, WSN can predict the probability and position of firing in coal goaf. In this paper, GtmWSN model and corresponding data transmitting algorithm, DTDGD, were proposed based on the "three-zone" theory. In forms of lifetimes, our design can meet the needs of goal temperature monitoring scenario and has practical guiding significance. Note that there may be interferences when GtmWSN nodes send data simultaneously; they are resolved by time division 


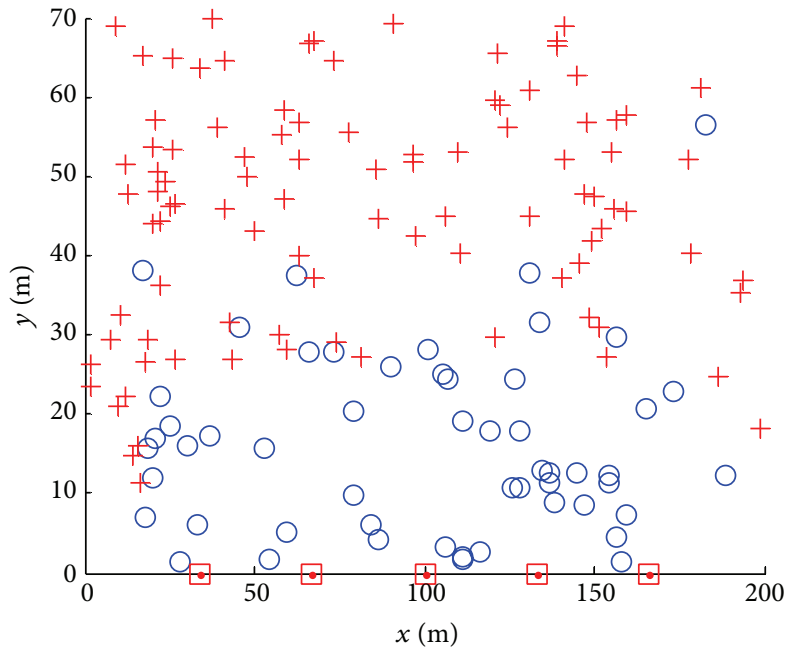

(a) $r=d s / 2+d s / 4$

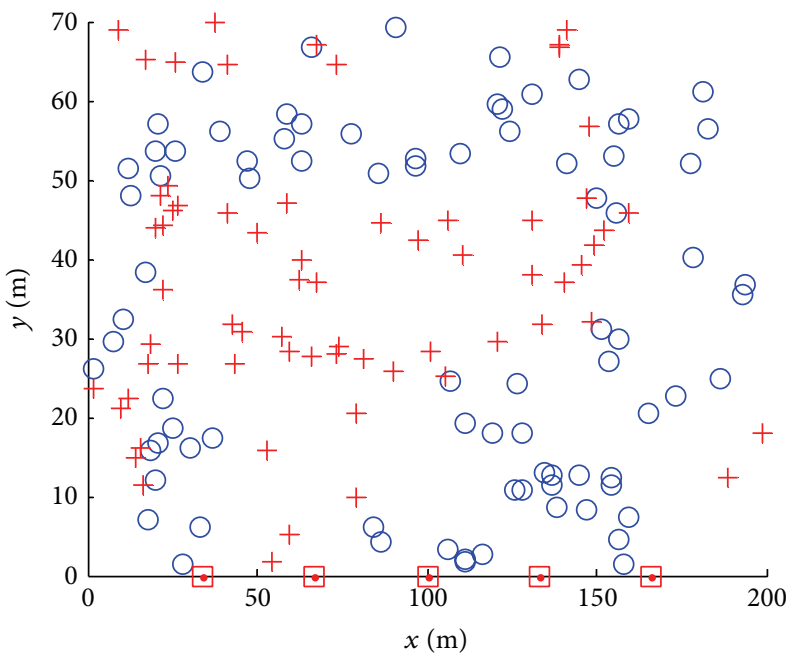

(c) $r=d s / 2+d s / 8$

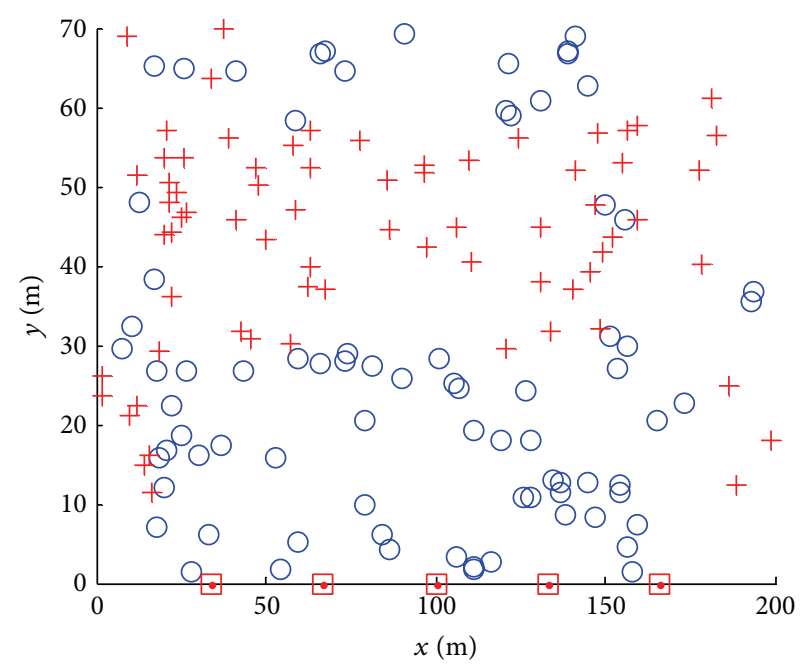

(b) $r=d s / 2+d s / 6$

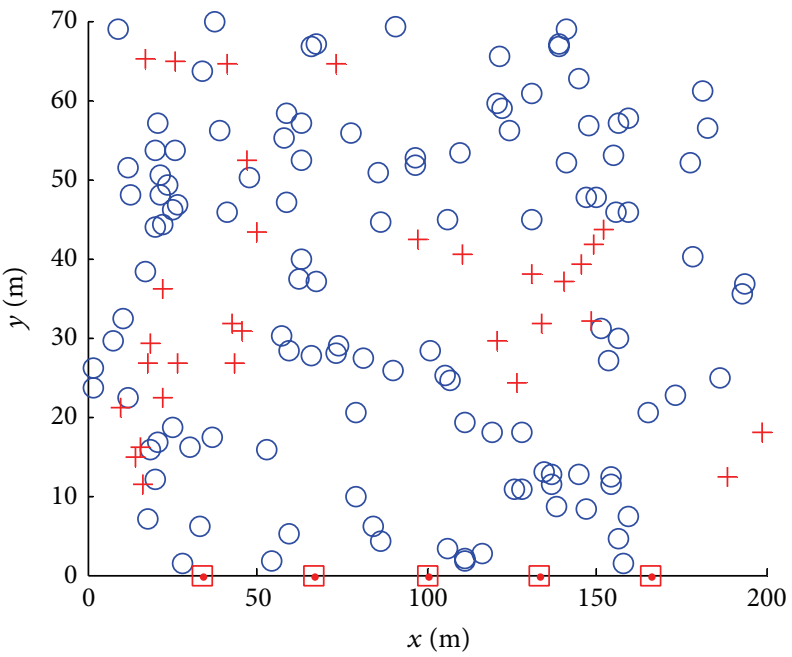

(d) $r=d s / 2+d s / 10$

FIGURE 11: Spatial distribution of dead nodes with different radii of virtual semicircle.

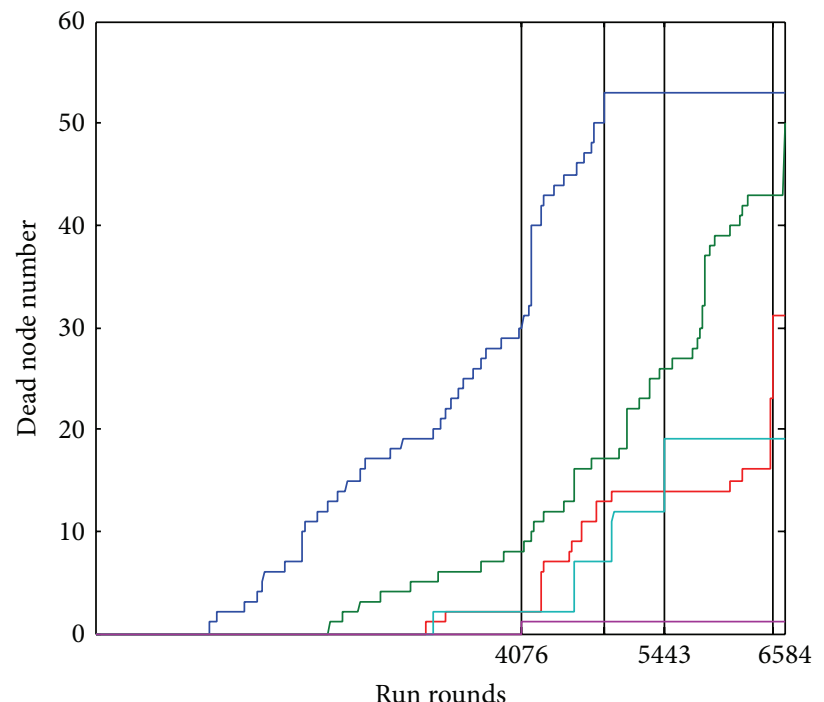

$\begin{aligned} \text { NumSink } & =3 \\ \text { NumSink } & =4 \\ \text { NumSink } & =5\end{aligned}$

- NumSink $=6$

FIGURE 12: Dead nodes number with different sink numbers. 


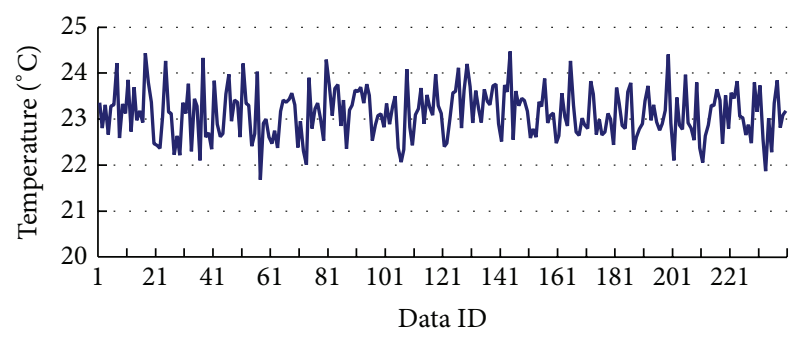

FIGURE 13: Temperature in two hours of 10302 working face.

in our simulation, and designing interference-resistant routing may be an appealing direction of future work.

\section{Conflict of Interests}

The authors declare that there is no conflict of interests regarding the publication of this paper.

\section{Acknowledgments}

The presented work was supported in part by the National Key Basic Research Program of China (2011CB707102), the National Natural Science Foundation of China (51204177), and special fund of National Higher-Education Institution General Research and Development project (2012BZB19).

\section{References}

[1] M. Y. Tang, Research on fire source positioning for goaf based on temperature field method [M.S. thesis], Anhui University of Science and Technology, 2005.

[2] Z. X. Li, Z. Y. Ti, and Y. J. Ji, "Numerical simulation of rise process in temperature field causedby nitrogen injection into goaf," China Safety Science Journal, vol. 15, no. 9, pp. 28-32, 2005.

[3] B. Tan, H. Y. Niu, C. N. He, and S. L. Feng, "Goaf coal spontaneous combustion temperature field theory and numerical analysis under mining conditions," Journal of Center South University (Science and Technology), vol. 44, no. 1, pp. 381-387, 2013.

[4] H. Q. Zhu, P. F. Liu, X. K. Liu, and M. Q. Yao, "Numerical simulation of temperature change and spontaneous combustion area in goaf during nitrogen injection process," ,Journal of Hunan University of Science \& Technology (Natural Science Edition), vol. 27, no. 1, pp. 1-6, 2012.

[5] L. An, E. J. Ding, and S. Q. Li, "Goaf wireless temperature monitoring system based on ZigBee technology," Transducer and Microsystem Technologies, vol. 31, no. 4, pp. 96-98, 2012.

[6] M. H. Anisi, A. H. Abdullah, S. A. Razak, and M. A. Ngadi, "An overview of data routing approaches for wireless sensor networks," Sensors, vol. 12, no. 4, pp. 3964-3996, 2012.

[7] Q. Liu, Research of wireless ad-hoc temperature measurement system used in goaf based on ZigBee [M.S. thesis], Xian University of Science and Technology, 2012.

[8] M.-G. Yu, X.-H. Chang, H.-L. Jia, and L.-X. Lu, "Analysis of spontaneous combustion "three-zone" in goaf based on Matlab," Meitan Xuebao/Journal of the China Coal Society, vol. 35, no. 4, pp. 600-604, 2010.
[9] T. X. Chu, S. Q. Yang, B. H. Yu, and Z. R. Zhang, "Research on "three-zone" of spontaneous combustion by measuring temperature and gas compositions in goaf," Express Information of Mining Industry, vol. 33, no. 9, pp. 42-45, 2008.

[10] E. Alotaibi and B. Mukherjee, "A survey on routing algorithms for wireless Ad-Hoc and mesh networks," Computer Networks, vol. 56, no. 2, pp. 940-965, 2012.

[11] X. M. Wang, J. P. Wang, K. J. Lu, and Y. L. Xu, "GKAR: a novel geographic K-anycast routing for wireless sensor networks," IEEE Transactions on Parallel and Distributed Systems, vol. 24, no. 5, pp. 916-925, 2013.

[12] J. Kim, X. Lin, and N. B. Shroff, "Optimal anycast technique for delay-sensitive energy-constrained asynchronous sensor networks," IEEE/ACM Transactions on Networking, vol. 19, no. 2, pp. 484-497, 2011.

[13] M. Won, W. Zhang, and R. Stoleru, "GOAL: a parsimonious geographic routing protocol for large scale sensor networks," Ad Hoc Networks, vol. 11, no. 4, pp. 453-472, 2013.

[14] Y. Yu, R. Govindan, and D. Estrin, "Geographical and energy aware routing: a recursive data dissemination protocol for wireless sensor networks," Tech. Rep. UCLA-CSD TR-010023, UCLA Comp.Sci. Dept., 2001.

[15] B. Leong, B. Liskov, and R. Morris, "Greedy virtual coordinates for geographic routing," in Proceedings of the 15th IEEE International Conference on Network Protocols (ICNP '07), pp. 71-80, October 2007.

[16] X. D. Yue, J. F. Wang, X. Q. Yuan, and Y. L. Hao, "Study of temperature evolution of spontaneous combustion area in goaf," Coal Technology, vol. 30, no. 3, pp. 107-109, 2011.

[17] S. Tao, A. L. Ananda, and M. C. Chan, "Greedy face routing with face identification support in wireless networks," Computer Networks, vol. 54, no. 18, pp. 3431-3448, 2010.

[18] S. K. Dhurandher, M. S. Obaidat, and M. Gupta, "An efficient technique for geocast region holes in underwater sensor networks and its performance evaluation," Simulation Modelling Practice and Theory, vol. 19, no. 9, pp. 2102-2116, 2011.

[19] Q. S. Hu, L. X. Wu, S. Zhang, and E. J. Ding, "Energy consumption and effecting factors of cooperative WSN routing algorithms," Journal of Huazhong University of Science \& Technology (Natural Science Edition), vol. 41, no. 2, pp. 81-85, 2013.

[20] D. X. Pan, Y. H. Wang, S. Q. Ma, B. Liu, X. F. Zhang, and Y. M. $\mathrm{Li}$, "Application of therm technology in fire prevention," Coal Engineering, vol. 53, no. 9, pp. 73-75, 2006.

[21] S. Q. Ma, J. F. Zhu, C. Wei, H. F. Liu, and C. J. Liu, "Applications of FBG technology on continuous monitoring of goal temperature," Coal Safety, vol. 12, no. 11, pp. 36-39, 2007. 


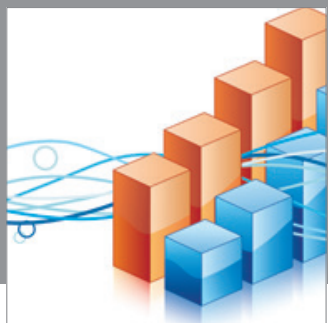

Advances in

Operations Research

mansans

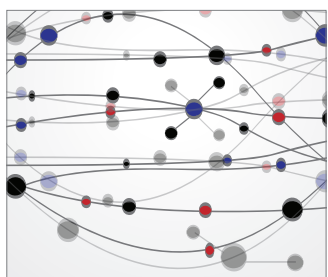

The Scientific World Journal
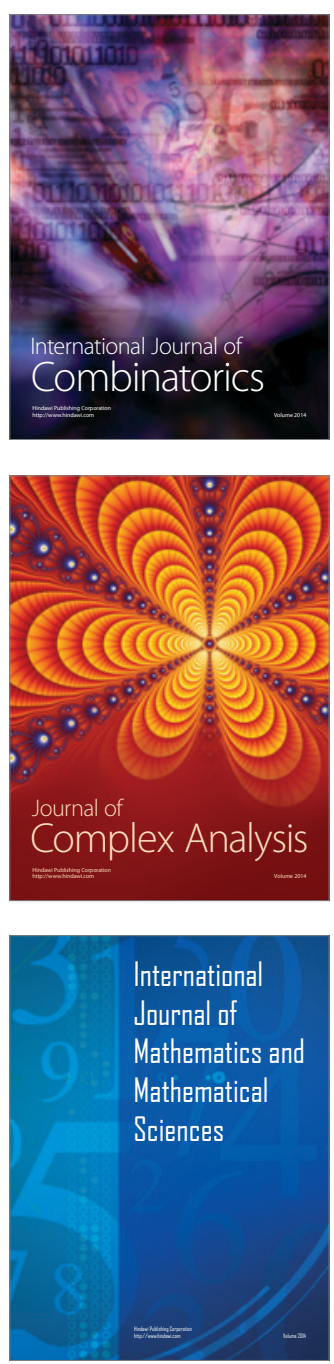
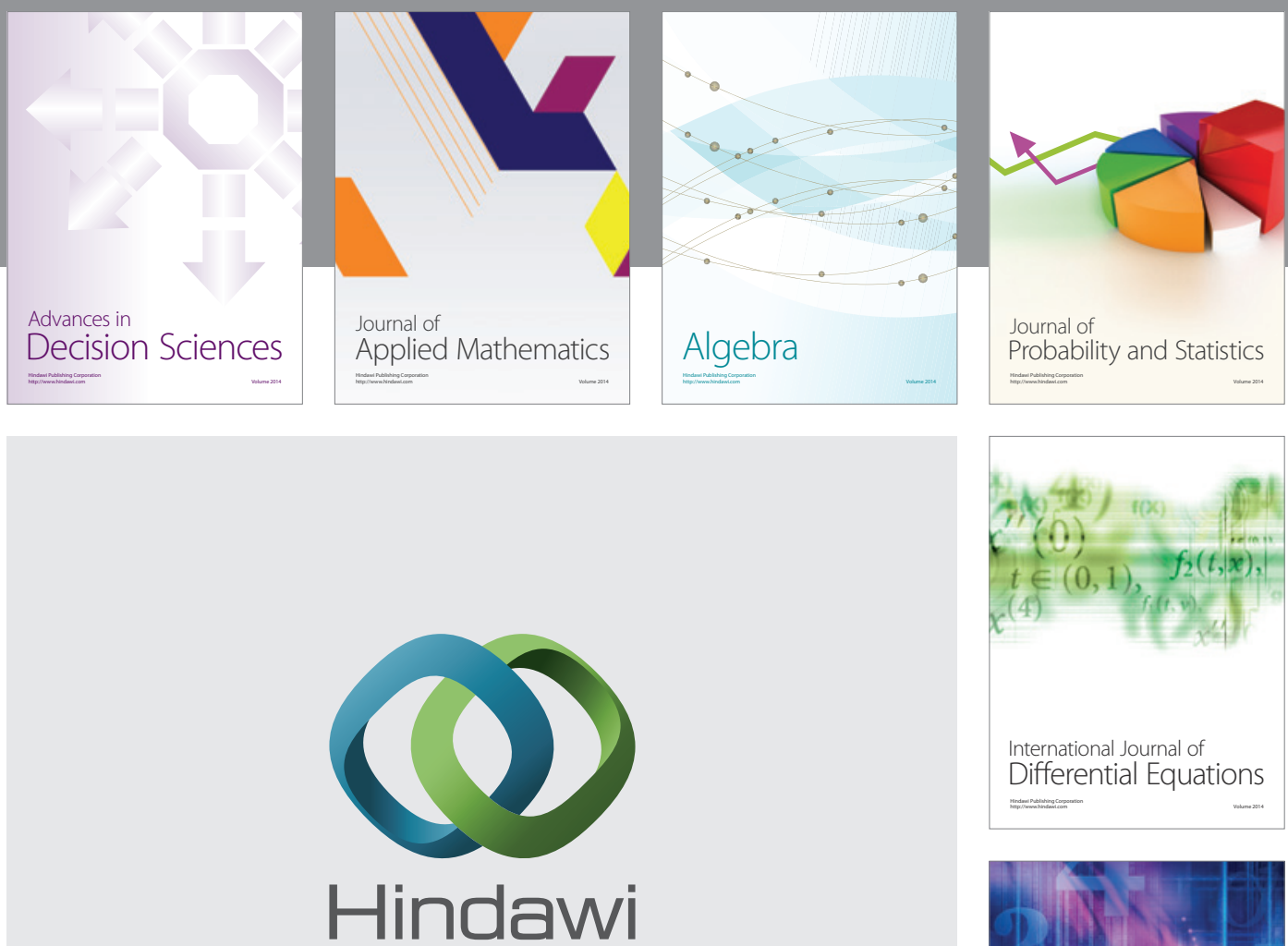

Submit your manuscripts at http://www.hindawi.com
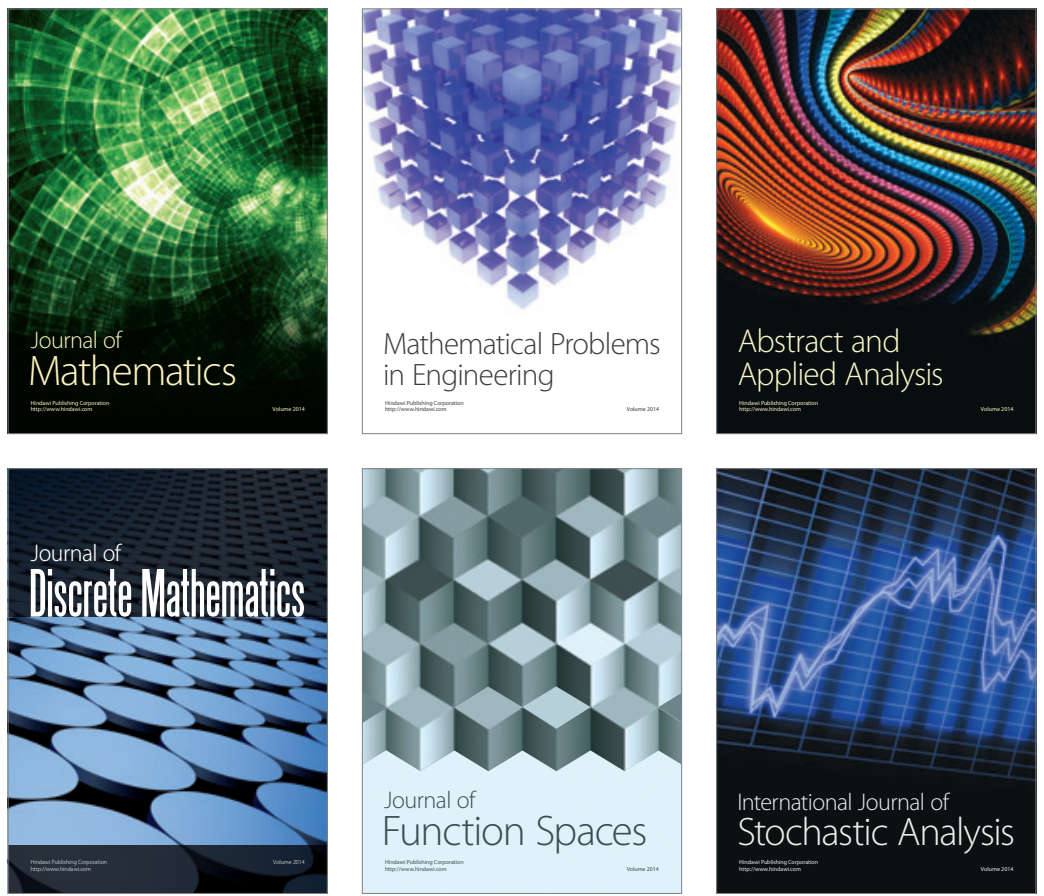

Journal of

Function Spaces

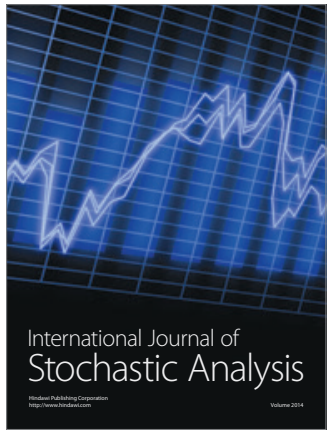

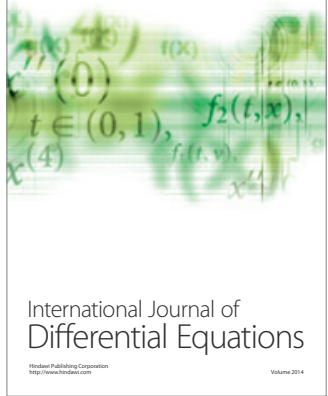
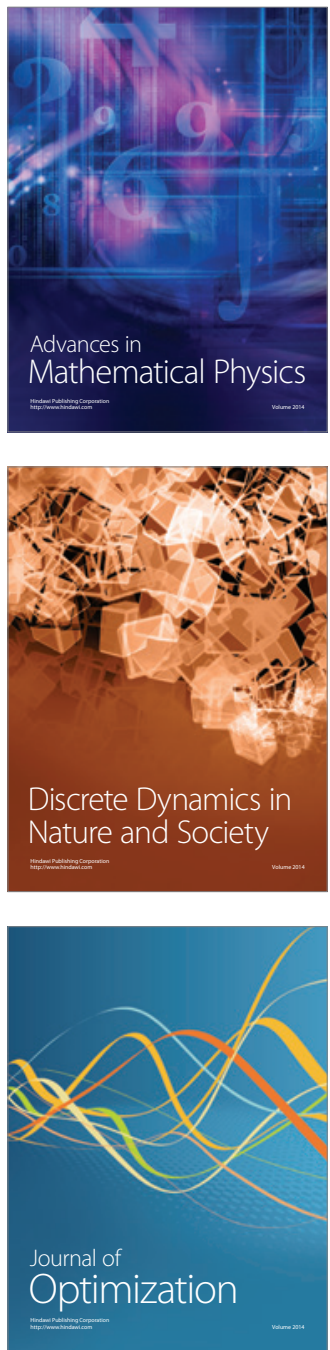